\title{
PREPARACIÓN DE SENSORES BASADOS EN ÓXIDO DE Fe DOPADOS CON Pt PARA LA DETECCIÓN DE METANO
}

\author{
Gino Picasso ${ }^{\text {a*}}$, Daniel Balboa ${ }^{\mathrm{a}}$, Rosario Sun-Kou ${ }^{\mathrm{b}}$
}

\begin{abstract}
RESUMEN
Sensores basados en nanopartículas de $\alpha-\mathrm{Fe}_{2} \mathrm{O}_{3}$ (hematita) puras y dopadas con $\mathrm{Pt}$ con cargas de 0,$1 ; 0,2$ y $0,3 \%$ (peso respecto del óxido) fueron preparados por el método de coprecipitación para la detección de metano, usando carbonato de sodio como agente precipitante y polietilenglicol (PEG) como antiaglomerante. Las muestras fueron caracterizadas por difracción de rayos $\mathrm{X}(\mathrm{XRD})$, sorción de $\mathrm{N}_{2}$ (método BET) y microscopía electrónica de transmisión (TEM). Los difractogramas de rayos $\mathrm{X}$ de las muestras han correspondido mayoritariamente a la fase $\alpha$-hematita. La introducción del Pt como dopante no ha modificado la estructura del óxido de partida. Todos los sensores han presentado isotermas micro-mesoporosas tipo IV. Según las micrografías TEM, las nanopartículas han sido heterogéneas con tamaños entre 50 a $150 \mathrm{~nm}$. Los tests catalíticos fueron realizados con concentraciones de 300 a 900 ppm de metano y temperaturas de trabajo de 150 a $400{ }^{\circ} \mathrm{C}$. El sensor con $0,3 \%$ de Pt y con $0,3 \%$ de PEG (20000) mostró la más alta señal de respuesta, para 900 ppm de metano a $300{ }^{\circ} \mathrm{C}$, debido probablemente a una buena formación de la fase cristalina, buena dispersión de las nanopartículas y por presentar la más alta superficie de contacto del material sensor.
\end{abstract}

Palabras clave: Sensores de gas, detección de metano, nanopartículas de $\mathrm{Pt} / \alpha-\mathrm{Fe}_{2} \mathrm{O}_{3}$.

\section{PREPARATION OF SENSORS BASED ON Fe OXIDE DOPED WITH Pt FOR DETECTION OF METHANE}

\begin{abstract}
Some sensors based on pure $\alpha-\mathrm{Fe}_{2} \mathrm{O}_{3}$ (hematite) and doped with \%Pt-loadings (respect to oxide) of 0,$1 ; 0,2$ y $0,3 \%$ have been prepared by precipitation method for detection of methane, sodium carbonate as precipitating agent and polyethylene glycol (PEG) as antiagglomeration agent. The samples were characterized by X-ray diffraction (XRD), sorption of $\mathrm{N}_{2}$ (BET method) and transmission electron microscopy (TEM). The XRD patterns showed that the crystalline structure of starting iron oxide has not been affected by the introduction of $\mathrm{Pt}$ as doping material. All the sensors depicted micro-mesoporous isotherms corresponding to type IV. TEM micrographs showed the average particle size of samples, ranging from 50 to $150 \mathrm{~nm}$. Sensor tests were performed in a range of concentrations from 300 to $900 \mathrm{ppm}$ and operation temperatures between 150 and $400{ }^{\circ} \mathrm{C}$. The sensor with $0,3 \%$ of $\mathrm{Pt}$ and $0,3 \%$ of PEG (20000) presented the highest gas response at $300^{\circ} \mathrm{C}$ with an initial concentration of $900 \mathrm{ppm}$ of methane probably due to the well-defined hematite crystalline structure, good dispersion of nanoparticles and the best contact surface over Pt surface.
\end{abstract}

Key words: Gas sensor, methane detection, nanoparticles of Pd-doped $\alpha-\mathrm{Fe}_{2} \mathrm{O}_{3}$.

\footnotetext{
a Laboratorio de Investigación de Fisicoquímica. Facultad de Ciencias. Universidad Nacional de Ingeniería. Av. Túpac Amaru 210. Rímac. Lima-Perú*gpicasso@uni.edu.pe.

b Departamento de Ciencias. Sección Química. Pontificia Universidad Católica del Perú. Avenida Universitaria 1801 - Lima 32
} 


\section{INTRODUCCIÓN}

Actualmente, en la comunidad científica existe una preocupación por la mejora de la calidad de vida, en particular, en la difusión de medidas preventivas para optimizar la calidad del ambiente. El uso de gas natural como fuente de energía, en lugar de leña de madera empleada en el pasado, ha permitido el uso más eficiente de los combustibles en las cocinas sin la formación de residuos sólidos y de especies intermedias de carbón ${ }^{1}$. Sin embargo, surgen nuevos retos en lo que respecta a la seguridad en la aplicación del combustible, para evitar posibles incendios producidos por los balones de gas debido a fugas, las cuales en pequeñas concentraciones, no detectables por el olfato del hombre, podrían ocasionar explosiones. Para evitar estos problemas se necesita de un sensor que registre fugas en pequeñas concentraciones, menores a $1000 \mathrm{ppm}$.

El óxido de hierro en su forma de hematita es un semiconductor tipo n, que detecta la presencia de gases reductores como metano, propano, amoniaco, por el cambio de conductividad eléctrica, debido a los mecanismos de oxidación superficial en los que se produce el intercambio de $\operatorname{cargas}^{2-6}$. Lamentablemente, este sensor es poco selectivo por sí solo; sin embargo, la adición de algún metal noble activo como el Pt, Pd y Ag ha permitido mejorar la selectividad del sensor hacia algunos gases, como $\mathrm{CO}^{7}, \mathrm{H}_{2} \mathrm{~S}^{8-11}$, debido a efectos electrónicos y químicos, que se refleja en una mejora de la sensibilidad y la reducción de las temperaturas de operación ${ }^{12-15}$.

El estudio de un gran número de óxidos semiconductores, incluyendo al óxido de hierro, permitió establecer que la respuesta eléctrica de éstos, en presencia de trazas de gases reactivos en aire, es un fenómeno común que no tiene carácter selectivo. En este sentido, el reducir el tamaño de las partículas del material sensor a escala nano ha sido una de las soluciones más extendidas para mejorar la selectividad y la señal de respuesta en la detección de gases, considerando la doble función del sensor: una función receptora que permite identificar una sustancia y una función transductora, que permite transformar esta señal química en una señal física medible y cuantificable. La señal física del transductor se difunde a través de la microestructura de los agregados de las nanopartículas del material sensor y se mide como resistencia del elemento cristalino ${ }^{12}$. El tamaño nanoparticulado del material sensor favorece la formación de los bordes de grano; de este modo, la transmisión de carga mejoraría sustancialmente. El problema es obtener un material nanoparticulado a base de óxidos semiconductores metálicos como la $\alpha$-hematita que posea una buena sensibilidad para detectar el gas de interés en un amplio rango de concentraciones y temperaturas ${ }^{13}$.

En un trabajo previo se estudió la mejora de las propiedades químicas y electrónicas de las nanopartículas de óxido de hierro mediante su dopaje con Pd para incrementar la sensibilidad del material para detectar propano a diferentes concentraciones y temperaturas de operación ${ }^{16}$. Se determinó que la sensibilidad en la detección de propano aumentó favorablemente para todos los sensores basados en hematita dopados con $\mathrm{Pd}$ respecto a su contraparte sin dopar, demostrando el efecto cooperante del Pd para incrementar la cantidad de oxígeno adsorbido sobre la superficie de las nanopartículas, mejorando la conductancia del material sensor. En otro trabajo, se estableció que el Pd jugó un rol sustancial en la señal 
de respuesta de los sensores basados en hematita en la detección de GLP ${ }^{17}$. La señal de respuesta creció linealmente con la concentración del gas y con la temperatura hasta los 300 ${ }^{\circ} \mathrm{C}$ y los tests de las señales de respuesta y reproducibilidad sugirieron la presencia de una fuerte influencia de la carga metálica del dopante. En la detección de metano se han reportado algunos trabajos usando óxido de $\mathrm{Sn}$ en forma de películas delgadas ${ }^{18-19}$, nanopolvos ${ }^{20}$, empleando nanopartículas de $\mathrm{Au}$ depositadas sobre filtros de $\mathrm{Fe}_{2} \mathrm{O}_{3}$ para el sensor $\mathrm{Pt} / \mathrm{SnO}_{2}{ }^{21}$. La aplicación de un sensor basado en hematita en la detección de metano todavía no se ha reportado, por esta razón, este trabajo es de interés por ser innovador y por el bajo coste del material de partida. En este trabajo, se estudia los efectos del tamaño molecular y del contenido del surfactante en las propiedades sensoras de la hematita dopada con Pt en la detección del metano; además, se ha explorado el efecto de la carga metálica del dopante, la dependencia de la temperatura y la concentración del gas en la señal de respuesta del sensor en un rango de concentraciones de 300 a 900 ppm del analito.

\section{Materiales de partida}

\section{PARTE EXPERIMENTAL}

Los materiales de partida fueron los siguientes:

- $\mathrm{Fe}\left(\mathrm{NO}_{3}\right)_{3} \cdot 9 \mathrm{H}_{2} \mathrm{O}_{(\mathrm{S})}($ Merck, 99,99\%).

- $\mathrm{H}_{2} \mathrm{PtCl}_{6} \cdot 6 \mathrm{H}_{2} \mathrm{O}_{(\mathrm{S})}(\mathrm{Merck}, 99,99 \%)$.

- $\mathrm{Na}_{2} \mathrm{CO}_{3(\mathrm{~S})}$ (Merck, 99,99\%).

- $\mathrm{HO}\left(\mathrm{C}_{2} \mathrm{H}_{4} \mathrm{O}\right)_{\mathrm{n}} \mathrm{H}_{(\mathrm{S})}(\mathrm{PEG}), \overline{\mathrm{m}}=2000,10000$ y $20000 \mathrm{~g} / \mathrm{mol}$ (Merck, 99,99\%).

\section{Preparación de los sensores}

Los sensores basados en óxidos de hierro fueron sintetizados en el Laboratorio de Investigación de Fisicoquímica de la Facultad de Ciencias de la Universidad Nacional de Ingeniería. Las diferentes muestras fueron preparadas mediante el método de coprecipitación, usando $\mathrm{Fe}\left(\mathrm{NO}_{3}\right)_{3} \cdot 9 \mathrm{H}_{2} \mathrm{O}_{(\mathrm{S})}$ y $\mathrm{H}_{2} \mathrm{PtCl}_{6} \cdot 6 \mathrm{H}_{2} \mathrm{O}_{(\mathrm{S})}$ como precursores, $\mathrm{Na}_{2} \mathrm{CO}_{3(\mathrm{~S})}$ como agente precipitante y polietilenglicol (PEG) como antiaglomerante o surfactante ${ }^{16-17}$. Se preparó soluciones de nitrato de hierro con diferentes concentraciones del dopante $\mathrm{Pt}$ (entre 0,1 a $0,3 \%$ respecto al peso de $\mathrm{Fe}_{2} \mathrm{O}_{3}$ ) y soluciones de carbonato de sodio $3 \mathrm{M}$ conteniendo diferentes concentraciones de surfactante (entre 0,1 a $0,3 \%$, calculada respecto al peso del carbonato). Para la preparación del sensor en general, se utilizó $250 \mathrm{~mL}$ de la solución de carbonato de sodio $3 \mathrm{M}$ con un porcentaje determinado de surfactante, a la cual se le adicionó $125 \mathrm{~mL}$ de nitrato de hierro, que contiene una cantidad prefijada de $\mathrm{Pt}$, a la temperatura de $80^{\circ} \mathrm{C}$ y a una velocidad de $0,5 \mathrm{~mL} / \mathrm{min}$. Adicionalmente, se adicionó a la mezcla carbonato de sodio $1 \mathrm{M}$ a una velocidad de $1 \mathrm{~mL} / \mathrm{min}$, para regular el $\mathrm{pH}$ hasta que se alcanzó un valor de $\mathrm{pH} \approx 10$. La mezcla óxido/hidróxido resultante fue filtrada al vacío. El sólido obtenido se secó en un horno a $110^{\circ} \mathrm{C}$ por $24 \mathrm{~h}$, luego se calcinó $400^{\circ} \mathrm{C}$ por $8 \mathrm{~h}$ en aire estático, empleando una rampa de calentamiento de $2^{\circ} \mathrm{C} / \mathrm{min}$. Finalmente, las muestras calcinadas se molieron y tamizaron hasta obtener partículas de tamaño igual o menor a $150 \mu \mathrm{m}$.

Las reacciones químicas y/o procesos físicos que ocurren en cada etapa se detallan a continuación:

Disolución: El precursor, sal metálica $\mathrm{Fe}\left(\mathrm{NO}_{3}\right)_{3} \cdot 9 \mathrm{H}_{2} \mathrm{O}$ tiene una constante dieléctrica alta $(78,5)$ que cuando se disuelve en agua se reduce las fuerzas electrostáticas entre los iones y 
$\mathrm{NO}_{3}$ de acuerdo a la ley de Coulomb. Adicionalmente, el agua hidrata a los iones $\mathrm{Fe}^{3+}$ por su naturaleza polar formando acuo-complejos:

$$
\left.\left[\mathrm{Fe}\left(\mathrm{NO}_{3}\right)_{3} \cdot 9 \mathrm{H}_{2} \mathrm{O}\right]_{(\mathrm{s})}+\mathrm{H}_{2} \mathrm{O}_{(\mathrm{l})} \longrightarrow\left[\mathrm{H}_{2} \mathrm{O}\right)_{5} \mathrm{Fe}-\mathrm{O}_{\mathrm{H}}^{\prime}\right]_{(\mathrm{ac})}^{\mathrm{H}} \quad \text { Ec. } 1
$$

Como la solución se polariza altamente, el agua se comporta como una base de Lewis, atacando al hidrógeno del enlace $\mathrm{O}-\mathrm{H}$ del ligando agua, promoviendo la desprotonación del acuo-complejo metálico y generando un complejo acuo-hidróxido, de acuerdo a la siguiente ecuación:

$$
[\left(\mathrm{H}_{2} \mathrm{O}\right)_{5} \mathrm{Fe}-\mathrm{O}-\overbrace{\mathrm{H}}]^{3+}+\left[\mathrm{Fe}(\mathrm{OH})\left(\mathrm{H}_{2} \mathrm{O}\right)_{5}\right]^{2+}+\mathrm{H}_{3} \mathrm{O}^{+} \quad \text { Ec. } 2
$$

Este mecanismo se basa en el modelo de la carga parcial propuesto por Livage ${ }^{22}$, que se sustenta en estudios teóricos sobre la electronegatividad realizados por Sanderson ${ }^{13}$, según los cuales el oxígeno del agua adquiere una carga parcial negativa $\delta(O)=-0,39$, mostrando un carácter nucleofílico, y el átomo de Fe del acuo-complejo presenta una alta carga parcial positiva $\delta(\mathrm{Fe})=+0,59$, la cual produce una fuerte polarización hacia la molécula de agua de su esfera de coordinación, y a la vez genera una carga positiva al hidrógeno, mediante una reacción ácido-base.

Precipitación: Al adicionar a la solución precursora la base (carbonato de sodio a $\mathrm{pH}=9,7$ ) se produce la precipitación del precursor. El mecanismo propuesto por Jolivet ${ }^{23}$, para un monómero se observa en la ecuación 3:

$$
\mid\left(\mathrm{H}_{2} \mathrm{O}\right)_{4}(\mathrm{OH}) \mathrm{Fe} \longrightarrow \mathrm{O}-\mathrm{H}^{2+}+2 \mathrm{OH}^{-} \longrightarrow\left[\mathrm{Fe}(\mathrm{OH})_{3}\left(\mathrm{OH}_{2}\right)_{3}\right]^{0}+2 \mathrm{H}_{2} \mathrm{O} \text { Ec. } 3
$$

Los cálculos en base al modelo de carga parcial muestran que los iones $\mathrm{OH}^{-}$actúan como bases de Lewis que atacan al hidrógeno de un ligando acuo del complejo; esta reacción ocurre dos veces hasta la formación del complejo de Fe de carga cero. El proceso de condensación puede ser generado mediante un mecanismo de sustitución nucleofílica de los grupos $\mathrm{OH}^{-}$ vecinos generando disociación de los grupos acuosos y condensación de los monómeros:
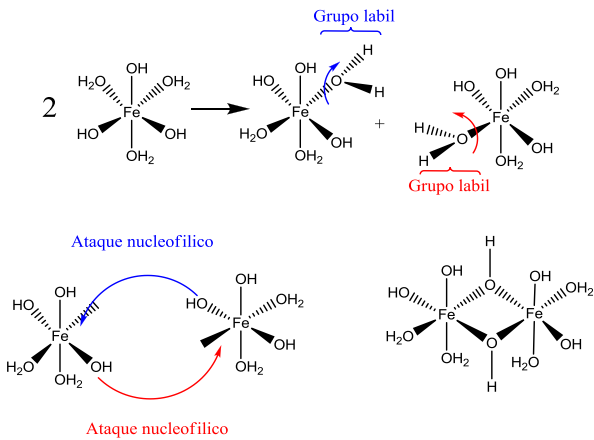
Secado: En este proceso se produce la vaporización de las moléculas de agua ligadas al acuo-complejo

$$
\mathrm{H}_{2} \mathrm{O}_{(\mathrm{l})} \rightarrow \mathrm{H}_{2} \mathrm{O}_{(\mathrm{g})} \quad \text { Ec. } 4
$$

Calcinación: La etapa de calcinación permitió obtener la especie $\alpha$-hematita por la deshidratación producida, que se evidenció por el análisis XRD.

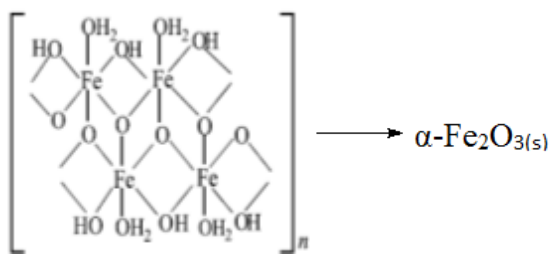

En la tabla 1 se muestra un resumen de las muestras preparadas en este trabajo.

Tabla 1. Lista de sensores preparados basados en $\alpha-\mathrm{Fe}_{2} \mathrm{O}_{3}$ variando tanto el tipo y el porcentaje de surfactante (PEG) y las cargas metálicas de Pt.

\begin{tabular}{|c|c|c|c|c|}
\hline \multirow{2}{*}{ Muestra } & \multirow{2}{*}{ Calcinación } & \multicolumn{2}{|c|}{ Surfactante (PEG) } & \multirow{2}{*}{$\% \mathrm{Pt}$} \\
\hline & & Tipo & $\%$ & \\
\hline $0,0 \mathrm{nc}$ & no & - & - & - \\
\hline 0,0 & sí & - & - & - \\
\hline $0,12 \mathrm{k} \mathrm{nc}$ & no & 2000 & 0,1 & - \\
\hline $0,1 \quad 2 \mathrm{k}$ & sí & 2000 & 0,1 & - \\
\hline $0,1 \quad 10 \mathrm{k}$ & sí & 10000 & 0,1 & - \\
\hline $0,120 \mathrm{k}$ & sí & 20000 & 0,1 & - \\
\hline $0,22 \mathrm{k}$ & sí & 2000 & 0,2 & - \\
\hline $0,210 \mathrm{k}$ & sí & 10000 & 0,2 & - \\
\hline $0,2 \quad 20 \mathrm{k}$ & sí & 20000 & 0,2 & - \\
\hline $0,32 \mathrm{k}$ & sí & 2000 & 0,3 & - \\
\hline $0,310 \mathrm{k}$ & sí & 10000 & 0,3 & - \\
\hline $0,320 \mathrm{k}$ & sí & 20000 & 0,3 & - \\
\hline $0,1 \mathrm{Pt}$ & sí & - & - & 0,1 \\
\hline $0,2 \mathrm{Pt}$ & sí & - & - & 0,2 \\
\hline $0,3 \mathrm{Pt}$ & sí & - & - & 0,3 \\
\hline $0,320 \mathrm{k} 0,1 \mathrm{Pt}$ & sí & 20000 & 0,3 & 0,1 \\
\hline $0,320 \mathrm{k} 0,2 \mathrm{Pt}$ & sí & 20000 & 0,3 & 0,2 \\
\hline $0,320 \mathrm{k} 0,3 \mathrm{Pt}$ & sí & 20000 & 0,3 & 0,3 \\
\hline
\end{tabular}


La nomenclatura empleada para las muestras fue la siguiente: el primer valor corresponde al porcentaje del surfactante, el segundo valor al tamaño molecular del surfactante dividido entre $1000 \mathrm{y}$ el tercer valor indica el porcentaje de Pt empleado. Ejemplo: 0,3 20k 0,3Pt representa la muestra que tiene $0,3 \%$ de surfactante con un tamaño molecular de 20000 y ha sido dopado con $0,3 \%$ de Pt. 00 - muestra de hematita sin surfactante y sin dopante.

\section{RESULTADOS Y DISCUSIÓN}

En este trabajo se ha realizado un estudio de los efectos de la calcinación, el porcentaje de surfactante y la carga metálica de Pt. Para cumplir los objetivos del trabajo, los sensores han sido caracterizados por las siguientes técnicas: sorción de $\mathrm{N}_{2}$ (método BET), difracción de rayos $\mathrm{X}(\mathrm{XRD})$ y microscopía electrónica de transmisión (TEM).

\section{A) Caracterización por difracción de rayos $\mathrm{X}$ (XRD)}

El análisis XRD se realizó en un equipo RIGAKU modelo Miniflex con tubo de cobre y filtro de níquel; los ensayos se realizaron con $30 \mathrm{kV}$ y $15 \mathrm{~mA}$; se utilizó un monocromador de grafito con el objetivo de seleccionar la radiación $\mathrm{CuK} \alpha$ del ánodo de cobre y se utilizó ángulos de barrido entre 5 y $100^{\circ}$ a una velocidad de $0,04 \%$ s.

\section{Efecto de la calcinación y del surfactante}

Como primer estudio se analizó el efecto de la temperatura de calcinación de la muestra. En la figura 1a se observa que los patrones de difracción en todos los sensores calcinados corresponden a $\alpha-\mathrm{Fe}_{2} \mathrm{O}_{3}$ (JCPDS - Internacional Centre for Diffraction Data $\mathrm{N}^{\mathrm{o}}$ 33-0664), mientras que en la muestras no calcinadas $0,0 n c$ y $0,12 \mathrm{k} n c$ no se observaron los dos picos mayoritarios de la hematita (a $33,18^{\circ}$ y $35,64^{\circ}$ ), correspondientes a los planos (104) y (110), respectivamente, debido posiblemente a que se formó una mezcla de óxidos hasta la temperatura de $80^{\circ} \mathrm{C}$. Por tanto, la calcinación a $400^{\circ} \mathrm{C}$ por $8 \mathrm{~h}$ fue imprescindible en la formación de la fase $\alpha$-hematita.

El efecto de la acción del surfactante en la estructura cristalina de los sensores se estudió variando el tipo de PEG (2000, 10000 y 20000) y el porcentaje de PEG añadido en la preparación del sensor. En la figura $1 \mathrm{~b}$ se observa que todas las muestras calcinadas pero con diferente tamaño molecular de PEG (2k, 10k y 20k) y a diferente concentración $(0,1 ; 0,2$ y $0,3 \%$ ) corresponden a la $\alpha$-hematita. Por tanto, no se ha observado una influencia apreciable del surfactante de $2 \mathrm{k}, 10 \mathrm{k}$ y $20 \mathrm{k}$ en la estructura de la hematita y la función del surfactante ha sido únicamente controlar el tamaño del grano.
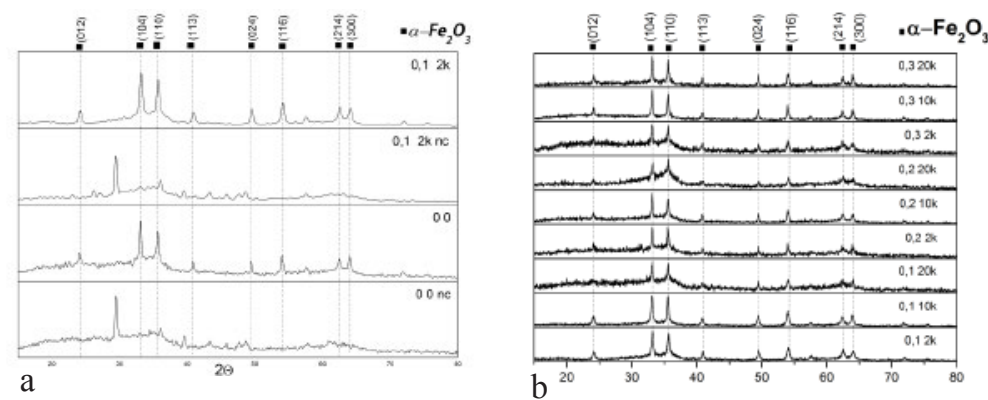

Figura 1. Comparación de los difractogramas XRD de las muestras calcinadas y no calcinadas (a) y de las muestras preparadas con diferente cantidad y porcentaje de PEG (b). 


\section{Efecto del material dopante}

Para analizar el efecto del material dopante $(\mathrm{Pt})$ en la estructura cristalina final, en la figura 2 se muestra en forma comparativa los difractogramas correspondientes a los sensores $0,1 \mathrm{Pt}$, $0,2 \mathrm{Pt}$ y $0,3 \mathrm{Pt}$, en ausencia de surfactante. Como se observa, el dopaje con Pt no ha afectado la estructura matriz del óxido de Fe de partida, ya que no se observa la presencia de picos característicos del Pt, debido probablemente a que el Pt se puede encontrar bastante disperso en la estructura externa del sensor, con excepción de la muestra $0,3 \% \mathrm{Pt}$, en la cual el metal dopante se introducido en la hematita, modificando su estructura.

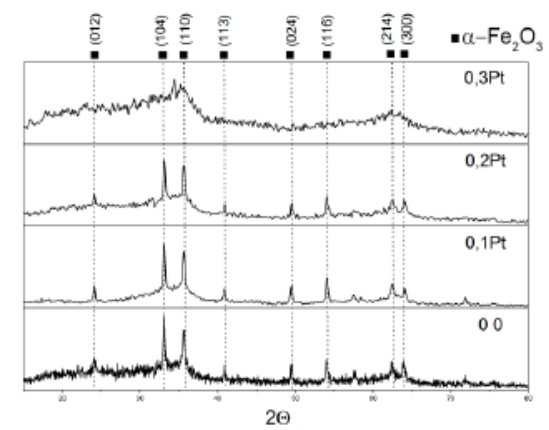

Figura 2. Difractogramas de las muestras preparadas con diferente carga del material dopante.

\section{B) Caracterización por sorción de $\mathrm{N}_{2}$ (método BET)}

Para la caracterización textural se utilizó el equipo Micromeritics GEMINI-VII serie t. Se utilizó $\mathrm{N}_{2}$ como gas de análisis $\left(\mathrm{N}_{2(\mathrm{~g})}, 99 \%\right)$ y nitrógeno líquido a $77 \mathrm{~K}$ como refrigerante. Previo a la adsorción, las muestras fueron desgasificadas a $250{ }^{\circ} \mathrm{C}$ por $2 \mathrm{~h}$. Los parámetros texturales se muestran en las columnas 4 y 5 de la tabla 2.

Tabla 2. Superficies específicas y tamaño medio de poro de diferentes sensores

\begin{tabular}{ccccc}
\hline Muestra & $\begin{array}{c}\text { \% surfactante } \\
\text { y tamaño } \\
\text { molecular }\end{array}$ & $\% \mathrm{Pt}$ & $\mathrm{S}_{\mathrm{BET}}\left(\mathrm{m}^{2} / \mathrm{g}\right)$ & $\begin{array}{c}\text { Tamaño } \\
\text { de poro } \\
(\mathrm{nm})\end{array}$ \\
\hline 0,0 & - & - & 56 & - \\
$0,32 \mathrm{k}$ & 0,$3 ; 2000$ & - & 83 & 43 \\
$0,310 \mathrm{k}$ & 0,$3 ; 10000$ & - & 121 & 34 \\
$0,320 \mathrm{k}$ & 0,$3 ; 20000$ & - & 153 & 25 \\
$0,1 \mathrm{Pt}$ & - & 0,1 & - & - \\
$0,2 \mathrm{Pt}$ & - & 0,2 & 58 & 38 \\
$0,3 \mathrm{Pt}$ & - & 0,3 & 63 & 22 \\
$0,320 \mathrm{k} 0,1 \mathrm{Pt}$ & 0,$3 ; 20000$ & 0,1 & 90 & 28 \\
0,3 20k 0,2Pt & 0,$3 ; 20000$ & 0,2 & 132 & 21 \\
0,3 20k 0,3Pt & 0,$3 ; 20000$ & 0,3 & 195 & 12 \\
\hline
\end{tabular}


En la figura 3 se presentan las isotermas de sorción del $\mathrm{N}_{2}$ de los sensores dopados con $\mathrm{Pt}$ y con PEG 20000 y los preparados únicamente con PEG (con diferente tamaño molecular), en ausencia de material dopante, para analizar el efecto de la carga metálica y el tamaño de la molécula del surfactante, respectivamente. Las isotermas de sorción de $\mathrm{N}_{2}$ obtenidas fueron de tipo IV, correspondientes a materiales micro-mesoporosos pero con una presencia mayoritaria de mesoporosidad. Los ciclos de histéresis en las muestras fueron del tipo $\mathrm{H} 3$, atribuibles a la formación de poros laminares. Se observa que la superficie específica aumentó con el incremento de la carga metálica de $\mathrm{Pt}$ (de 90 a $195 \mathrm{~m}^{2} / \mathrm{g}$ ) y con el aumento del tamaño molecular del surfactante (de 83 a $153 \mathrm{~m}^{2} / \mathrm{g}$ ). En el primer caso, es posible que el $\mathrm{Pt}$ en presencia del surfactante ocupe con preferencia la superficie externa del sensor y el segundo caso, se deba, probablemente, a efectos estéricos que presenta la cadena que rodea al grano, limitando su crecimiento y aumentando los espacios entre ellos.
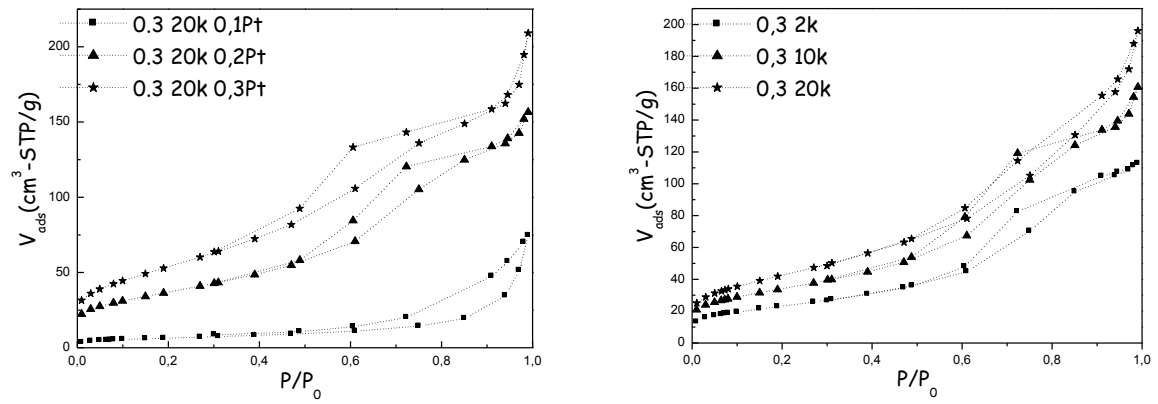

Figura 3. Isotermas de sorción de $\mathrm{N}_{2}$ de los sensores dopados con Pt (izquierda) y sin dopar pero con surfactante de diferente tamaño molecular (derecha).

\section{C) Caracterización por microscopía electrónica de transmisión (TEM)}

El microscopio electrónico de transmisión emite un haz de electrones dirigido hacia el objeto que se desea analizar. Una parte de los electrones se retrodispersan o son absorbidos por la muestra y otros lo atraviesan formando una imagen aumentada de la muestra; dicha imagen se registra mediante una placa fotográfica. El microscopio electrónico de transmisión (TEM) empleado en este trabajo es del modelo Philips EM 300G de $80 \mathrm{keV}$, que tiene un límite de resolución de aproximadamente $2 \mathrm{~nm}$. El protocolo experimental de preparación fue el siguiente: La muestra en suspensión fue dispersada mediante ultrasonido; posteriormente, con una pipeta Pasteur, se colocó una gota de esta suspensión sobre una rejilla con membrana como soporte y se mantuvo así hasta que el solvente se evaporó completamente.

En las figuras 4 y 5 se presenta las micrografías comparativas del sensor a base de hematita (sin dopar y sin surfactante) con el análogo (con surfactante) y del sensor 0,3 $0,1 \mathrm{Pt}$ (sin surfactante) con su contraparte $0,30,2 \mathrm{k} 0,1 \mathrm{Pt}$ el (con surfactante). Como se puede apreciar, los tamaños de las nanopartículas, en ambos casos, disminuyeron en promedio de 50 a 30 $\mathrm{nm}$ en el sensor de hematita con surfactante, comparado con su análogo sin surfactante, mostrando el efecto favorable del surfactante en la disminución del tamaño de la partícula. Los tamaños medio de las partículas obtenidas han sido heterogéneos en el rango de 50 a $150 \mathrm{~nm}$. 

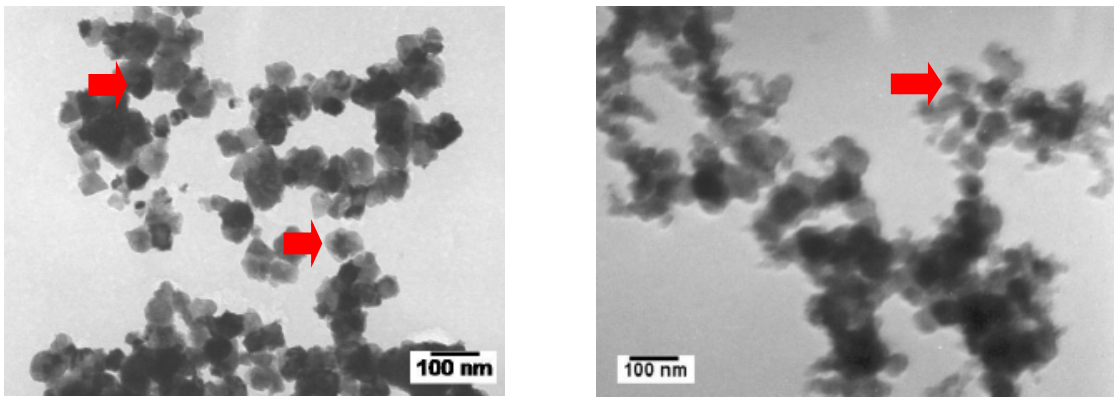

Figura 4. Micrografías TEM de la hematita sin dopar, sin surfactante (izquierda), con surfactante (derecha).
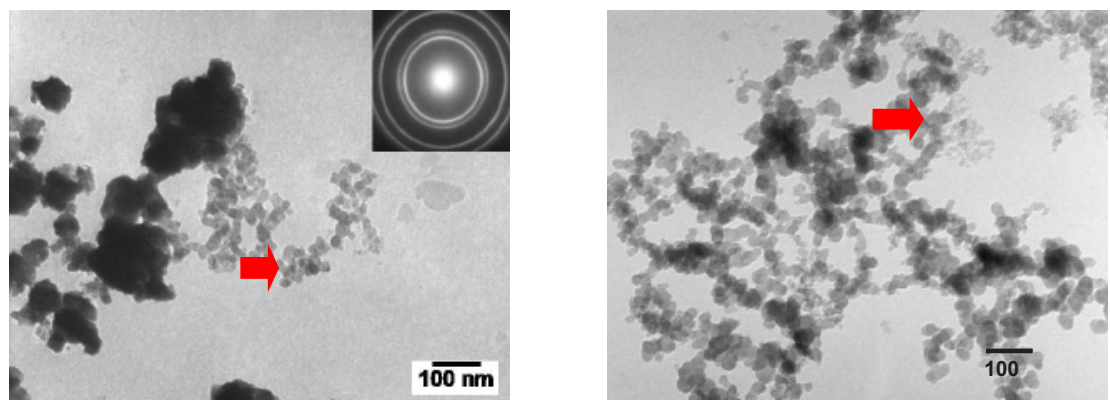

Figura 5. Micrografías TEM del sensor $0,30,1 \mathrm{Pt}$ sin surfactante (izquierda) y con su similar 0,3 $0,2 \mathrm{~K} 0,1 \mathrm{Pt}$, con surfactante (derecha).

\section{Medición de la señal de respuesta de los sensores}

Los sensores de gas preparados en este trabajo son dispositivos basados en hematita dopados con Pt que actúan como semiconductores tipo-n, que presentan el interés adicional de su posible miniaturización e integración que permite conseguir dispositivos complejos capaces de detectar bajos niveles de concentración del gas ${ }^{13}$. Los sensores han sido depositados en un substrato de alúmina, similares a los empleados en trabajo previo ${ }^{17}$.

Para realizar los tests de detección de metano con los sensores preparados se utilizó un gas patrón ( $1 \% \mathrm{CH}_{4}$ en $\mathrm{N}_{2}$, LINDE) y aire comprimido como diluyente para obtener las mezclas gaseosas seleccionadas en el rango de 300 a 900 ppm. Las temperaturas de operación de los sensores en este trabajo fueron en el rango de 150 a $400 \mathrm{C}^{\circ}$. Estas variaciones se realizaron con el objetivo de determinar la temperatura óptima de trabajo de cada sensor a diferentes concentraciones y para evaluar la influencia del porcentaje de Pt en la señal de respuesta del sensor (S), que se determinó mediante la siguiente expresión: $\mathrm{G}_{\mathrm{gas}} / \mathrm{G}_{0}$, donde $\mathrm{G}_{\mathrm{gas}}$, representa la conductancia en presencia del gas metano mezclado con aire y $\mathrm{G}_{0}$, es la conductancia en presencia sólo de aire. A través del equipo de ensayo de sensores se registran los valores de voltaje; estos valores eléctricos son transformados a valores de conductancia mediante la 
siguiente fórmula: $\mathrm{G}=\mathrm{V} /\left[\mathrm{R}\left(\mathrm{V}_{\mathrm{r}}-\mathrm{V}\right)\right]$, donde, $\mathrm{V}$ es el voltaje que se mide en el equipo, $\mathrm{V}_{\mathrm{r}}$ es el voltaje de referencia ( 5 voltios), $R$ es igual a $20 \mathrm{Mohm}$. Previo a los tests se calibró el controlador de temperaturas y se estableció los caudales de flujo en los flujómetros para la obtención de la concentración de metano en aire.

\section{Efecto de la carga metálica del dopante}

Se ha estudiado el efecto del material dopante sin presencia de surfactante para comprobar el efecto intrínseco del Pt. Los sensores dopados con Pt han mostrado tener mayor señal de respuesta comparada con la muestra de óxido de Fe (sin dopar), independiente de la temperatura de operación, indicando el efecto cooperativo del Pt para la promoción de la sensibilidad de detección del metano (figura 6). La mayor señal de respuesta se obtuvo con el sensor dopado con $0,3 \%$ Pt seguido de las muestras conteniendo $0,2 \%$ y $0,1 \%$ Pt. Este efecto se puede explicar por la mayor superficie específica que tiene el sensor con mayor contenido de Pt (tabla 2) y mejor estructura cristalina (figura 2). Algunos autores sugieren que el Pt actúa en el sensor por un mecanismo químico, que resulta de una reacción química superficial ${ }^{12}$. Este mecanismo sugiere que el Pt forma clusters superficiales que actúan como sitios activos para la adsorción preferente del metano, facilitando su interacción con el oxígeno quimisorbido en la superficie del óxido de Fe. La máxima señal de respuesta en las muestras dopadas y con $700 \mathrm{ppm}$ de metano se obtuvo a la temperatura de $300^{\circ} \mathrm{C}$; dicha temperatura fue mayor que la obtenida con los mismos sensores en contacto con $300 \mathrm{ppm}$ de metano; ello debido, probablemente, al efecto de la concentración de metano que a mayor temperatura facilitó la migración de las especies adsorbidas superficiales.

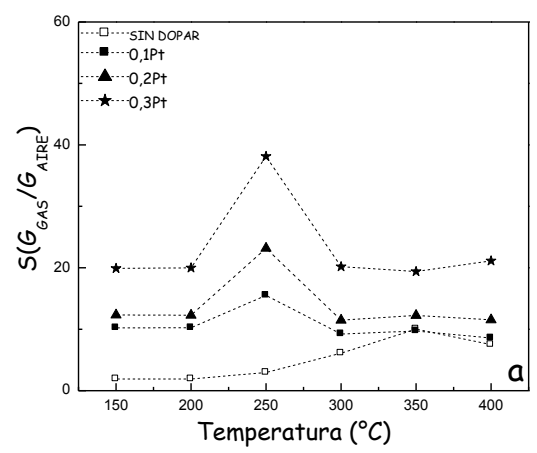

(a)

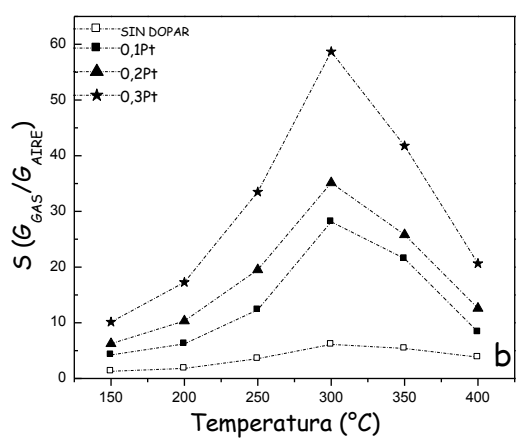

(b)

Figura 6. Efecto del contenido de material dopante en la señal de respuesta de los sensores con la concentración inicial de metano de: 300 ppm (a) y 700ppm (b). 


\section{Efecto del tamaño molecular del surfactante en ausencia de dopante}

En la figura 7 se muestra el efecto de la concentración y tamaño molecular del surfactante en la señal de respuesta de los sensores, en ausencia de Pt, considerando una concentración inicial de metano de 300 ppm y 700 ppm. En todos los casos se observó que independiente del tamaño y concentración del surfactante, la temperatura de máxima sensibilidad fue de $300^{\circ} \mathrm{C}$ y la señal de respuesta del sensor mejoró con el incremento del tamaño molecular del surfactante. Para un mismo surfactante, la señal del sensor mejoró con el aumento de la concentración del mismo. La mejor señal se obtuvo con el sensor $0,3,20 \mathrm{k}$ con una carga del $0,3 \%$ respecto al óxido de Fe solo. Los efectos del tamaño molecular del surfactante mejoraron la dispersión de las fases, al disminuir el tamaño medio de la nanopartícula (figura 5) y al aumentar de la superficie específica (tabla 2), por ejemplo, la señal de respuesta para la muestra $0,320 \mathrm{k}$ con $153 \mathrm{~m}^{2} / \mathrm{g}$ es más intensa que la obtenida con el sensor $0,32 \mathrm{k}$ con 83 $\mathrm{m}^{2} / \mathrm{g}$. El aumento de la concentración inicial del analito mejoró también la señal de respuesta, por ejemplo, para el sensor $0,320 \mathrm{k}$ a $300^{\circ} \mathrm{C}$ trabajando con concentraciones iniciales de metano de 300 y 700 ppm la señal obtenida fue de 30 y 50, respectivamente.
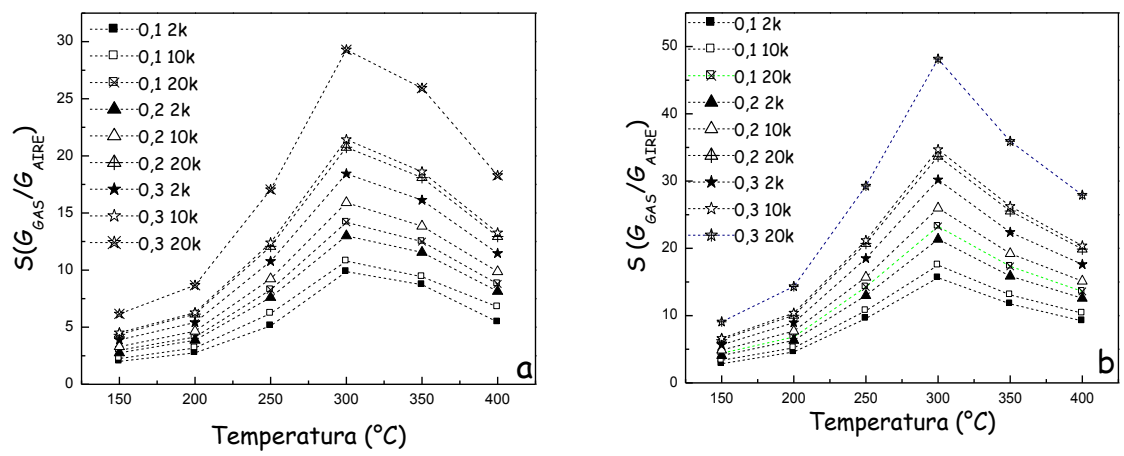

Figura 7. Efecto del tamaño molecular del surfactante en la señal de respuesta de los sensores (sin dopante) con una concentración inicial de metano de: 300 ppm (a) y 700 ppm (b).

\section{Efecto de la carga metálica del dopante en presencia del surfactante}

En la figura 8 se presentan los resultados del efecto del contenido de material dopante $(\% \mathrm{Pt})$ en presencia de surfactante con masa molecular de 20000, en la señal de respuesta del sensor, considerando una concentración inicial de metano de 300 y 700 ppm. La tendencia en la señal de respuesta fue similar a los resultados observados cuando se varió sólo la carga metálica (figura 6); sin embargo, se puede apreciar que en ambos casos, la señal de respuesta del sensor mejoró ostensiblemente al sumar los efectos favorables del dopante y del surfactante. En la figura 9a se muestra el valor comparativo de las señales de respuesta de los mejores sensores obtenidos considerado el efecto del surfactante $(0,320 \mathrm{k})$, el efecto individual del dopante $(0,3 \mathrm{Pt})$ y el efecto conjunto del surfactante y dopante $(0,320 \mathrm{~K} 0,3 \mathrm{Pt})$. La mejor respuesta fue la obtenida con el sensor $0,320 \mathrm{~K} 0,3 \mathrm{Pt}$; este resultado se puede atribuir a que su área superficial $\left(195 \mathrm{~m}^{2} / \mathrm{g}\right)$ fue la más alta obtenida entre todos los sensores (tabla 2) y a la mejor dispersión de sus nanopartículas observada por la técnica de microscopía electrónica de transmisión (figura 5) con una buena estructura cristalina (figura 2). Finalmente, los sensores 
han mostrado una buena reproducibilidad de la señal de respuesta (figura 9b), como se puede apreciar para el sensor $0,320 \mathrm{~K}$ 0,3Pt; esto también representa una buena opción para su posible aplicación en la detección de metano a concentraciones diferentes a las estudiadas en este trabajo (100 a 900 ppm).
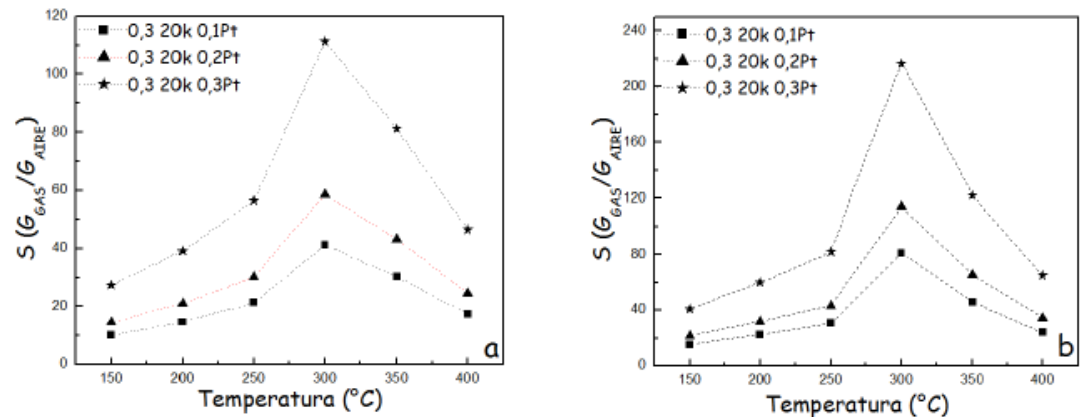

Figura 8. Efecto del contenido de material dopante en presencia de surfactante con masa molecular de 20000, en la señal de respuesta de los sensores con una concentración inicial de metano de 300 ppm, (a) y 700 ppm (b).
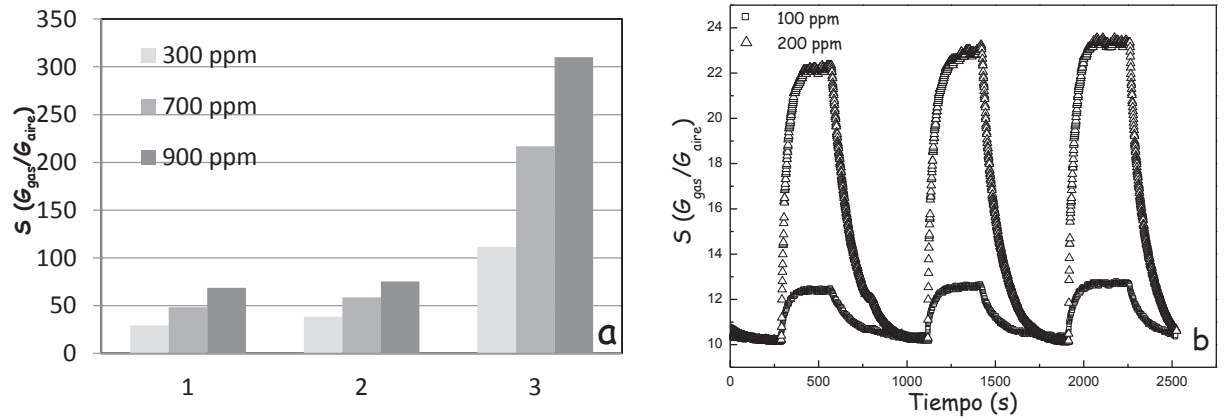

Figura 9. Señales de respuesta de los sensores $0,320 \mathrm{k}(1), 0,3 \mathrm{Pt}(2), 0,320 \mathrm{k} \mathrm{0,3 \textrm {Pt }}$ (3) para diferentes concentraciones iniciales de metano (a). Reproducibilidad de la señal de respuesta para el sensor 0,3 20k 0,3Pt (b).

\section{CONCLUSIONES}

El análisis XRD de los sensores basados en nanopartículas de $\alpha-\mathrm{Fe}_{2} \mathrm{O}_{3}$ puros y dopados con Pt por coprecipitación, mostraron la presencia mayoritaria de la fase hematita en todos los materiales, presentando mayor cristalinidad las muestras dopadas con Pt. El análisis TEM ha mostrado la presencia de una fuerte dispersión de nanopartículas con un tamaño de medio de 50 a $150 \mathrm{~nm}$. La superficie específica aumentó con el contenido de Pt y de surfactante. El aumento del tamaño molecular del surfactante, PEG y del contenido de Pt, mejoró la señal de respuesta final del sensor, siendo el mejor el 0,3\% 20k, respecto a los otros sensores.

Los tests realizados a diferentes temperaturas de operación y concentraciones iniciales del metano, con los sensores conteniendo Pt y surfactante, revelaron que la señal de respuesta 
de los sensores tuvo una fuerte influencia de la carga del sensor y del tamaño y cantidad de surfactante usado en la preparación de las muestras. La señal mejoró aún más con el aumento de la concentración inicial de metano. El sensor 0,3 20k 0,3Pt presentó el mejor comportamiento a la temperatura de $300^{\circ} \mathrm{C}$ en el rango de concentraciones iniciales de metano de 300 a 900 ppm, debido, probablemente, a un mayor valor de la superficie específica, mejor dispersión de las nanopartículas y una buena formación cristalina de la fase activa (hematita). El sensor mostró también una buena reproducibilidad en la señal de respuesta que lo hace adecuado para su aplicación como sensor a bajas concentraciones de metano.

\section{AGRADECIMIENTO}

Los autores agradecen al Instituto de Investigación de la Universidad Nacional de Ingeniería (IGI) por el proyecto IGI - 2012-II por el apoyo logístico para la preparación de las muestras y para la realización de los tests de medición de las señales de respuesta de los sensores.

\section{BIBLIOGRAFÍA}

1. Wetchakun K., Samerjai T., Tamaekong N., Liewhiran C., Siriwong C., Kruefu V., Wisitsoraat A., Semiconducting metal oxides as sensors for environmentally hazardous gases, Sens. Actuators B 2011; 160: 580- 591.

2. Oliveira Luiz C.A., Zaera F., Lee I, Lima D.Q., Ramalho T.C., Silva A.C, Fonseca E.M.B., Nb-doped hematites for decomposition of isopropanol: Evidence of surface reactivity by in situ CO adsorption, Appl. Catal. A 2009; 368(1-2):17-21.

3. Jadhav V.V., Patil S.A., Shinde D.V., Waghmare S. D., Zate M.K., Mane R.S., Han S.H., Hematite nanostructures: Morphology-mediated liquefied petroleum gas sensors, Sensor Actuator B 2013; 188: 669-674.

4. Cuong D.N., Hoa T.T., Khieu Q.D., Hoa N.D., Hieu V.N., Gas sensor based on nanoporous hematite nanoparticles: Effect of synthesis pathways on morphology and gas sensing properties, Curr. Appl. Phys. 2012; 12(5): 1355-1360.

5. $\quad \mathrm{Su}$ D., Kim H.S., Kim W.S., Wang G., Synthesis of tuneable porous hematites $(\alpha-\mathrm{Fe} 2 \mathrm{O} 3)$ for gas sensing and lithium storage in lithium ion batteries, Micropor. Mesopor. Mat. 2012; 149(1): 36-45.

6. Cui X., Liu T., Zhang Z., Wang L., Zuo S., Zhu W., Hematite nanorods with tunable porous structure: Facile hydrothermal-calcination route synthesis, optical and photocatalytic propertie, Powder Technol. 2014; 266: 113-119.

7. Jiang X.C., Yu A.B., Synthesis of $\mathrm{Pd} / \alpha-\mathrm{Fe} 2 \mathrm{O} 3$ nanocomposites for catalytic CO oxidation, J. Mater. Process. Technol. 2009; 209 (9): 4558-4562.

8. Hussain S., Maqsood A., Structural and electrical properties of Pb-doped Sr-hexa ferrites, J. Alloys Compd. 2008; 466 (1-2): 293-298.

9. Wang Y., Wang S., Zhao Y, Zhu B., Kong F., Wang D., Wu S., Huang W., Zhang S., H2S sensing characteristics of Pt-doped thick sensors, Sens. Actuators B 2007; 125(1): 79-84.

10. Wang Y., Kong F., Zhu B., Wang S., Wu S., Huang W., Synthesis and characterization of $\mathrm{Pd}$-doped $\alpha-\mathrm{Fe}_{2} \mathrm{O}_{3} \mathrm{H}_{2} \mathrm{~S}$ sensor with low power consumption, Mater. Sci. Eng. B 2007; 140(1-2):98-102.

11. Wang Y., Wang Y., Cao J., Kong F., Xia H., Zhang J., Zhu B., Wang S., Wu S., Low- 
temperature $\mathrm{H} 2 \mathrm{~S}$ sensors based on Ag-doped $\alpha$-Fe2O3 nanoparticles, Sens. Actuators B 2008; 131(1): 183-189.

12. Yamazoe N., New approaches for improving semiconductor gas sensors, Sens. Actuators B 1991; 5(1-4): 7-19.

13. Franke M.E., Koplin T.J., Simon U., Metal and metal oxide nanoparticles in chemiresistors: does the nanoscale matter, Small 2006; 2: 36-50.

14. Neri G., Bonavita A., Ipsale S., Rizzo G., Baratto C., Faglia G., Sberveglieri G., Pd- and Ca-doped iron oxide for ethanol vapor sensing, Mater. Sci. Eng. B 2007; 139(1):41-47.

15. Seino S., Kinoshita T., Otome Y., Nakagawa T., Okitsu K., Mizukoshi Y., Nakayama T., Sekino T., Niihara K., Yamamoto T.A., Gamma-ray synthesis of magnetic nanocarrier composed of gold and magnetic iron oxide, J. Magn. Magn. Mater. 2005; 293(1):144-150.

16. Picasso, G., Sun-Kou, M.R., Lagos Araujo L., Rojas Barreto J., Preparación y empleo de nanopartículas de $\alpha-\mathrm{Fe}_{2} \mathrm{O}_{3}$ como sensores para la detección de propano, Revista ION 2012; 25(1): 35-41.

17. Picasso G., Sun-Kou M.R., Vargasmachuca O., Rojas J., Zavala C., Lopez A., Irusta S., Sensors based on porous Pd-doped hematite $\left(\alpha-\mathrm{Fe}_{2} \mathrm{O}_{3}\right)$ for LPG detection, Micropor. Mesopor. Mat. 2014; 185: 79-85.

18. Bose S., Chakraborty S., Ghosh B.K., Dasc D., Sena A., Maiti H.S., Methane sensitivity of Fe-doped $\mathrm{SnO}_{2}$ thick films, Sens. Actuators B 2005; 105(2): 346-350.

19. Abbaszadeha D., Ghasempoura R., Rahimi F., Iraji Z. A., Effective factors on methane sensing of tin-oxide activated by palladium in sol-gel process, Sens. Transducers J. 2006; 73: 819-825.

20. Cabot A., Vila A., Morante J.R., Analysis of the catalytic activity and electrical characteristics of different modified $\mathrm{SnO} 2$ layers for gas sensors, Sens. Actuators $B$ 2002; 84(1):12-20.

21. Oliaee S.N., Khodadadi A. Mortazavi Y., Alipour S., Highly selective Pt/SnO2 sensor to propane or methane in presence of $\mathrm{CO}$ and ethanol, using gold nanoparticles on $\mathrm{Fe}_{2} \mathrm{O}_{3}$ catalytic filter. Sensors and Actuators B 2010; 147(2-3): 400-405.

22. Livage J., Henry M., Sanchez C., Sol-gel chemistry of transition metal oxide, Ed. Laboratoire du Chemie de la matiere condense, Universite Pierre et Marie Curie, Paris, France, 1989.

23. Jolivet, M. Henry, J. Livage, Metal Oxide Chemistry and Synthesis, Universite Pierre et Marie Curie, Paris, France, 1989. 